\title{
Tests of a novel method to assay SNM using polarized photofission and its sensitivity in the presence of shielding
}

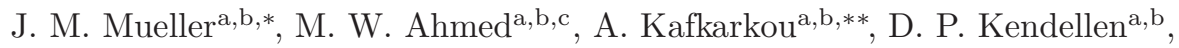 \\ M. H. Sikora ${ }^{\mathrm{a}, \mathrm{d}}$, M. C. Spraker ${ }^{\mathrm{e}}$, H. R. Weller ${ }^{\mathrm{a}, \mathrm{b}}$, W. R. Zimmerman ${ }^{\mathrm{a}, \mathrm{b}}$ \\ ${ }^{a}$ Triangle Universities Nuclear Laboratory, Durham, NC 27710, USA \\ ${ }^{b}$ Department of Physics, Duke University, Durham, NC 27708, USA \\ ${ }^{c}$ Department of Mathematics and Physics, North Carolina Central University, Durham, NC \\ 2770\%, USA \\ ${ }^{d}$ Department of Physics, George Washington University, Washington, DC 20052, USA \\ ${ }^{e}$ Department of Physics, University of North Georgia, Dahlonega, GA 30597, USA
}

12

A novel method to identify Special Nuclear Material was recently developed 1]. This method relies upon using a linearly polarized $\gamma$-ray beam to induce photofission of a sample and then comparing the prompt fission neutron yields in and out of the plane of beam polarization. The present paper will describe experimental tests of this new technique and assess its sensitivity in the presence of shielding. The capability of this technique to measure the enrichment of uranium was tested by using combinations of thin ${ }^{235} \mathrm{U}$ and ${ }^{238} \mathrm{U}$ foils of known enrichments. The sensitivity of this assay to shielding by lead, steel, and polyethylene was experimentally measured and simulated using GEANT4. These tests show that the measured asymmetry can indeed be used to determine the enrichment of materials composed of an admixture of ${ }^{235} \mathrm{U}$ and ${ }^{238} \mathrm{U}$, and this asymmetry is relatively insensitive to moderate amounts of shielding.

Keywords: Active interrogation; Fission; Enrichment; Assay; Polarized

beams; Neutron detectors

\section{Introduction}

Non-destructively identifying the presence of shielded Special Nuclear Material (SNM), or attempting to measure the enrichment of SNM, is sufficiently

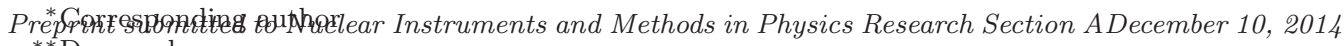
** Deceased

Email address: mueller@tunl.duke.edu (J. M. Mueller) 
difficult that many potential solutions have been proposed. These solutions are typically divided into passive measurements, which rely on the natural radioactivity of the material of interest, and active measurements which use a beam to interrogate a sample. Many solutions which might work for one subset of SNM, such as ${ }^{235} \mathrm{U}$ and ${ }^{238} \mathrm{U}$, may not work for other types of SNM, such as plutonium isotopes.

In passive measurements, natural emissions from a sample are used to detect or assay SNM. These natural emissions could be $\alpha$ particles or $\gamma$ rays from $\alpha$ decay, or neutrons or $\gamma$ rays from spontaneous fission. Many passive techniques have been developed to detect $\gamma$ rays following $\alpha$-decay to measure the enrichment of ${ }^{235,238} \mathrm{U}\left[2\right.$, 3], 44, 5] and ${ }^{239,240} \mathrm{Pu}$ [6]. However, it may be difficult to use these techniques to measure the enrichment of samples composed of an admixture of ${ }^{233} \mathrm{U}$ and ${ }^{232} \mathrm{Th}$ because of the long half-life of ${ }^{232} \mathrm{Th}$ and the absence of $\gamma$ rays emitted from its decay. Passive measurements of $\alpha$-particles produced by $\alpha$-decay could be used to assay samples of ${ }^{232} \mathrm{Th}$ and ${ }^{233,235,238} \mathrm{U}$ [7]. Unfortunately, this technique is more difficult to use with ${ }^{239,240} \mathrm{Pu}$ since their $\alpha$-particle decay energies are very close. There is, however, some progress on unfolding measured spectra to assay plutonium samples [7, 8]. Passive measurements of $\alpha$ particles are also complicated by the fact that the $\alpha$ particles will straggle or stop in the sample itself and in any casing or shielding, so sample preparation is typically required. Another possible passive measurement to identify ${ }^{240} \mathrm{Pu}$ takes advantage of its relatively short spontaneous fission half-life and looks for spontaneous prompt fission neutrons. These neutrons are sufficient to discover samples of ${ }^{240} \mathrm{Pu}$, but the spontaneous fission half-lives of ${ }^{235,238} \mathrm{U}$ are long enough that this technique is less practical to identify these nuclides [9].

In active measurements, a beam is used to interrogate a sample. This beam is typically composed of either neutrons or high-energy $\gamma$ rays [10]. Thermal neutrons, either moderated at the neutron generator or moderated within the sample, are a particularly sensitive probe of the presence of fissile nuclear material. After interrogating the sample with thermal neutrons, one could detect either prompt neutrons such as with the differential die-away technique [11, 12] 
or delayed $\gamma$ rays [13] to identify the presence of fissile material. However, active interrogation with thermal neutrons is not sensitive to the presence of non-fissile but fissionable material such as ${ }^{238} \mathrm{U}$ or ${ }^{232} \mathrm{Th}$. Therefore active interrogation with thermal neutrons cannot be easily used to measure the enrichment or isotopics of SNM. Fast neutron beams are sensitive to the presence of fissile and non-fissile materials, and Ref. [14] has found that ${ }^{232} \mathrm{Th},{ }^{237} \mathrm{~Np}$, and ${ }^{238} \mathrm{U}$ could be distinguished by detecting differences in the yields of specific delayed $\gamma$ rays. These differences fundamentally arise from variations in the mass distribution of the daughters following fission, so when the fission mass distributions are similar, such as for ${ }^{237} \mathrm{~Np}$ and ${ }^{238} \mathrm{U}$, the sensitivity of this technique decreases [14].

Active measurements with $\gamma$-ray beams can also be used to measure the enrichment or isotopics of a sample. Delayed $\gamma$ rays from fission could be used to measure the isotopics of samples in a technique similar to that of Ref. 14], but this again becomes more difficult when the fission product mass distributions are similar. Nuclear Resonance Fluorescence (NRF) in principle is the most sensitive technique, since in principle it could be used to quantify the amount of each nuclide present in a sample of SNM [15, 16]. The NRF technique seems to be the most versatile method to distinguish different isotopes in SNM, though it is still under development.

A new technique has recently been proposed to measure the fissile versus non-fissile, but fissionable, content of SNM [1]. This technique relies upon a 6 $\mathrm{MeV}$, linearly polarized $\gamma$-ray beam to induce fission of actinide nuclei within a sample. Ref. [17] demonstrated that the relative number of prompt neutrons in the plane of beam polarization compared to those perpendicular to the plane of beam polarization varies depending on the fissile nature of the sample. For non-fissile samples, such as natural uranium or thorium, a factor of roughly 2-3 more prompt neutrons are emitted parallel to the plane of beam polarization compared to those emitted perpendicular to the plane of beam polarization. However, for fissile samples, such as nearly pure ${ }^{235} \mathrm{U}$ or ${ }^{239} \mathrm{Pu}$, about the same neutron yield is observed in and out of the plane of beam polarization. 
One advantage of this proposed technique is its generality. The fundamental cause of this polarization asymmetry in the prompt neutrons is the even-A or odd-A character of the fissioning nuclide. With a $6 \mathrm{MeV} \gamma$-ray beam, we expect that all even-A nuclei should have a significant polarization asymmetry, and all odd-A nuclei should have almost no polarization asymmetry. The even-A nuclei in general are not fissile, while the odd-A nuclei generally are either fissile or are otherwise of special interest, such as ${ }^{237} \mathrm{~Np}$. Therefore, this technique might be able to measure the fissile versus non-fissile content of an arbitrary mix of actinide isotopes.

However, before that potential is considered, additional experiments must be performed to assess the viability of this technique. No previous work has ever attempted to use the polarization asymmetry to measure the fissile versus non-fissile content of SNM. In the present paper, we report measurements of the polarization asymmetry for combined foils of ${ }^{235} \mathrm{U}$ and ${ }^{238} \mathrm{U}$ as direct tests of the ability of this technique to determine the enrichment of the combined foils. In particular, this could be of interest as a new way to distinguish natural or reactor-grade uranium from weapons-grade uranium.

Additionally, photofission has been considered as a method to identify clandestinely transported shielded SNM in an active interrogation environment. By measuring the polarization asymmetry of the prompt neutrons, in principle one could not only identify the presence of shielded SNM, but also simultaneously measure the enrichment of the shielded SNM. However, this capability depends on how shielding affects the prompt neutron polarization asymmetry. We report here novel measurements of the dependence of the polarization asymmetry on the thickness and orientation of common shielding materials: high density polyethylene, steel, and lead.

\section{Description of the experiment}

The experimental techniques used were similar to those in Refs. [1, 17, 18], so only a short description of the experimental setup and analysis is provided here. 
A nearly $100 \%$ linearly-polarized, high-intensity $\left(\sim 10^{7} \gamma / \mathrm{s}\right)$, pulsed $\gamma$-ray beam was generated using the High Intensity $\gamma$-Ray Source $(\mathrm{HI} \gamma \mathrm{S})$ located at Duke University [19, 20]. The $\gamma$-ray beam was created by colliding a high-power Free Electron Laser (FEL) beam with an electron beam in a storage ring, and a quasimonoenergetic $\gamma$-ray beam of $6.1 \mathrm{MeV}$ was generated. The energy spread of the $\gamma$-ray beam was approximately $3 \%$ (FWHM). The beam was pulsed at a rate of $5.58 \mathrm{MHz}$, and the duration of each beam pulse was significantly shorter than our timing resolution. This beam repetition rate, with 179 ns between beam bursts, is well suited to fast neutron detection using time of flight methods.

At the $\mathrm{HI} \gamma \mathrm{S}$ facility, there are two ways to produce linearly polarized $\gamma$ ray beams: using either horizontal undulators, or using a technique similar to that described in Ref. [21]. In previous experiments [1, 17, 18], the FEL was generated using the first method. This technique of producing an FEL allowed for the generation of well-collimated $\gamma$-ray beams with nearly $100 \%$ polarization. Therefore, in our previous work, the $\gamma$-ray beam polarization was assumed to be $100 \%$ with no systematic uncertainty. This technique of polarized $\gamma$-ray beam production was used for the present shielding measurements with lead, steel, and some of the measurements with polyethylene.

In the rest of the experiments presented here, such as the tests with multiple target foils and the other shielding measurements with polyethylene, a new method of FEL production was used to generate linearly polarized $\gamma$-ray beams. This new technique is conceptually similar to Ref. [21], where a buncher magnet was used to controllably switch the beam polarization. The resulting $\gamma$-ray beam was found to be highly polarized, but the polarization was not longer consistent with $100 \%$. In the work described here, the $\gamma$-ray beam polarization was continuously monitored with a separate experimental setup upstream of the actinide target. The polarimeter consisted of a $\mathrm{D}_{2} \mathrm{O}$ target and four liquid scintillator detectors filled with $\mathrm{BC}-501 \mathrm{~A}$. The $\mathrm{D}_{2} \mathrm{O}$ was kept in a $5.03 \mathrm{~cm}$ long and $3.18 \mathrm{~cm}$ diameter cell composed of thin plastic walls. The neutron detectors were $12.70 \mathrm{~cm}$ in diameter and $5.08 \mathrm{~cm}$ thick. The outgoing neutrons from the $d(\gamma, n)$ reaction were detected by the liquid scintillators. Two detectors were 
placed in the horizontal plane at a scattering angle of $90^{\circ}$, and the other two detectors were placed in the vertical plane at a scattering angle of $90^{\circ}$. The $\mathrm{d}(\gamma, \mathrm{n})$ reaction has a large polarization asymmetry in the outgoing neutrons for beam energies below $20 \mathrm{MeV}$, as indicated by theoretical calculations [22, 23, 24, 25] and experimental measurements [26, 27, 28, 29]. At a beam energy of $6.1 \mathrm{MeV}$, the predicted polarization asymmetry for the $\mathrm{d}(\gamma, \mathrm{n})$ reaction at a scattering angle of $90^{\circ}$ in a pointlike geometry is 0.972 according to Ref. [23, 30]. According to Ref. [25, 31], the asymmetry is 0.966 with a calculated uncertainty of approximately 1\%. Because of the good agreement between these two predictions, 0.972 has been used as the theoretical polarization asymmetry, and a systematic uncertainty of $1 \%$ was assumed. Based on this prediction and a GEANT4 [32] simulation, the linear polarization of the beam was determined to range from 96.5 to $98.0 \%$, with a statistical uncertainty of less than $0.2 \%$ per measurement and a global systematic uncertainty of $1.0 \%$ across all measurements. The systematic uncertainty is dominated by the uncertainty assigned to the theoretical prediction of the $\mathrm{d}(\gamma, \mathrm{n})$ asymmetry.

After traversing the $\mathrm{D}_{2} \mathrm{O}$ cell, the $\gamma$-ray beam was incident on a set of uranium foils located several meters downstream of the $\mathrm{D}_{2} \mathrm{O}$ cell. A set of ${ }^{235} \mathrm{U}$ and ${ }^{238} \mathrm{U}$ foils was used to test the enrichment measurement capabilities of our aforementioned technique [1]. The three ${ }^{235} \mathrm{U}$ foils each had thicknesses of approximately $0.24 \mathrm{~g} / \mathrm{cm}^{2}$, masses of approximately $1.5 \mathrm{~g}$, and enrichments of $93.7 \%$. The different ${ }^{238} \mathrm{U}$ foils were approximately $0.11,0.40$, and $1.18 \mathrm{~g} / \mathrm{cm}^{2}$ thick with masses of $0.72 \mathrm{~g}, 1.5 \mathrm{~g}$, and $6.16 \mathrm{~g}$ respectively and enrichments of $0.7 \%$ (natural uranium).

Some of the uranium foils were visibly nonuniform, so it was not possible to calculate the enrichment of the combined foils by simply measuring their thicknesses. Therefore, the enrichment of each foil combination was determined by the measured counting rates. For example, one of the ${ }^{238} \mathrm{U}$ foils would be inserted into the beam, and the count rate relative to the $\mathrm{D}_{2} \mathrm{O}$ polarization monitor was measured. Then, without moving the ${ }^{238} \mathrm{U}$ foil, a ${ }^{235} \mathrm{U}$ foil was placed approximately $1 \mathrm{~cm}$ downstream of the ${ }^{238} \mathrm{U}$ foil, and the polarization 
asymmetry and the new count rate relative to the polarization monitor was measured. The count rate due to the ${ }^{235} \mathrm{U}$ foil was calculated by subtracting off the measured pure ${ }^{238} \mathrm{U}$ count rate. The measured ${ }^{235} \mathrm{U}$ and ${ }^{238} \mathrm{U}$ count rates, weighted by the photofission cross sections $\left(\sigma_{f}\right)$ and average prompt neutron multiplicities $(\bar{\nu})$ for each isotope, were proportional to the amount of ${ }^{235} \mathrm{U}$ and ${ }^{238} \mathrm{U}$ in the beam. Previous measurements have found the ratio of $\sigma_{f} \bar{\nu}$ for ${ }^{235} \mathrm{U}$ to that of ${ }^{238} \mathrm{U}$ to be $1.02 \pm 0.09$ at a beam energy of $6.1 \mathrm{MeV}[33,34]$. The uncertainty in this value was treated as a systematic uncertainty. In addition to this systematic uncertainty in the enrichment, there was also a statistical uncertainty present in the measured enrichment from the statistical uncertainty in each measured counting rate.

A series of measurements was also performed using only ${ }^{238} \mathrm{U}$ foils and various shielding materials. For these measurements, the combined thickness of the ${ }^{238} \mathrm{U}$ foils was approximately $6.9 \mathrm{~g} / \mathrm{cm}^{2}$. One shielding test was performed with a $2.45 \mathrm{~cm}$ thick copper block inserted about $45 \mathrm{~m}$ upstream of the target. This distance was chosen to significantly reduce the probability of scattered $\gamma$ rays reaching the target, simply from the small solid angle of the target as viewed from the block. The copper block was located upstream of the primary $\gamma$-ray beam collimator, which removed any large-angle scattered $\gamma$ rays from the copper block and prevented them from reaching the neutron detectors. Another series of tests was performed using blocks of lead, steel, and high density polyethylene. In these tests, each block was $7.62 \mathrm{~cm}$ tall, $7.62 \mathrm{~cm}$ wide, and $2.54 \mathrm{~cm}$ thick. Figure 1 shows the different shielding scenarios tested with these shielding materials. First, one block of each type was inserted approximately 2 cm directly upstream of the uranium foil. This type of shielding will be referred to as "F" for "front" shielding. The beam had to pass through the $2.54 \mathrm{~cm}$ thick side of the block to reach the target. Then, four blocks of each type of shielding material were placed around the uranium target to shield the target from the neutron detectors. These blocks were again located approximately 2 $\mathrm{cm}$ from the target and completely shielded the target from all of the neutron detectors. The neutrons had to pass through the $2.54 \mathrm{~cm}$ thick side of the block 


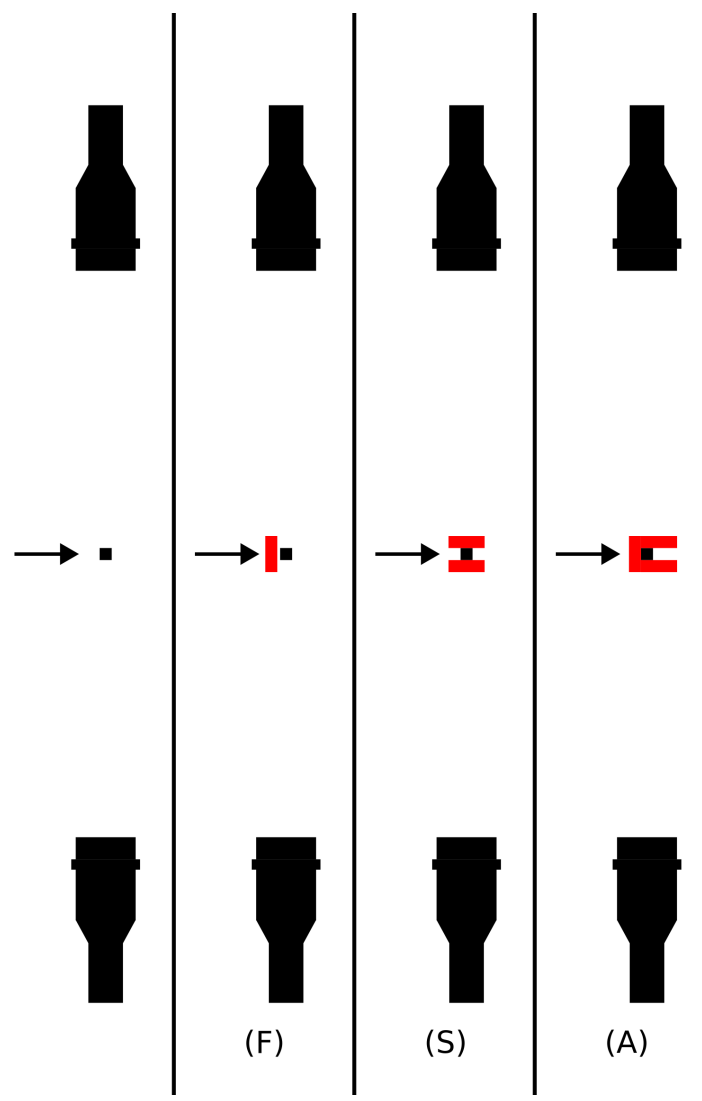

Figure 1: The different shielding scenarios with lead, steel, and high density polyethylene blocks are shown. The leftmost illustration is without any shielding materials. The $\gamma$-ray beam is indicated by the arrow and is incident on the target. The following illustration, indicated by (F) for front shielding, shows a shielding block (red) in front of the target. The next illustration, labeled (S) for side shielding, shows shielding blocks between the target and neutron detectors. The final schematic, labeled (A) for all shielding, shows shielding blocks in front of and on the sides of the target. Shielding materials and neutron detectors above and below the plane of the figure are in the same configuration and are omitted for clarity. The illustration is approximately to scale except for the target which was enlarged for the figure.

to reach a detector. This scenario will be referred to as "S" for "side" shielding. Finally, the front and side shielding scenarios were combined so that the front face and four sides of the target were shielded with $2.54 \mathrm{~cm}$ of each shielding material. This configuration is called "A" for "all" shielding. All of these tests 
were performed using the original method of polarized $\gamma$-ray beam production.

As a final shielding test using only high density polyethylene, the amount of shielding on the sides of the target was varied. Measurements were taken with $2.54,5.08,10.16,15.24$, and $20.32 \mathrm{~cm}$ of high density polyethylene shielding on the sides of the target. The shielding began approximately $4 \mathrm{~cm}$ from the target. In these cases, blocks of various dimensions were used in order to completely shield the target. However, the width of each block was always $15.24 \mathrm{~cm}$. This width was chosen to ensure the $12.70 \mathrm{~cm}$ diameter detector was shielded by the polyethylene. All of these tests were performed using the new method of polarized $\gamma$-ray beam production.

All of the experimental setups described above used the same neutron detection method. The prompt fission neutron yields were measured using four liquid scintillator detectors filled with BC-501A. These detectors had the same dimensions as the $\mathrm{BC}-501 \mathrm{~A}$ detectors used in the $\mathrm{D}_{2} \mathrm{O}$ polarimeter. Two detectors were placed in the horizontal plane at a scattering angle of $90^{\circ}$, and the other two detectors were placed in the vertical plane at a scattering angle of $90^{\circ}$. The detectors were placed approximately $70 \mathrm{~cm}$ from the target. Pulse shape discrimination was used to eliminate $\gamma$-ray backgrounds. The energies of detected neutrons were determined based on their time-of-flight, and neutron energy thresholds were set at $\mathrm{E}_{n}=1.5 \mathrm{MeV}$. This threshold was chosen to eliminate potential backgrounds from the $(\gamma, \mathrm{n})$ reaction. The neutron backgrounds from delayed fission neutrons from earlier events were subtracted from the prompt neutron events by measuring the yield out-of-time with the beam pulse. The background-subtracted prompt fission neutron yield was integrated over all neutron energies above the energy threshold, and the polarization asymmetry $\Sigma$ at a scattering angle of $90^{\circ}$ :

$$
\Sigma\left(90^{\circ}\right)=\frac{1}{P} \frac{L+R-U-D}{L+R+U+D}
$$

was measured. Here $L$ is the prompt neutron yield in the beam left detector, $R$ is the yield in the beam right detector, $U$ is the yield in the beam up detector, and $D$ is the yield in the beam down detector. The fractional beam polarization 
$P$ was measured for each run independently using the $\mathrm{D}_{2} \mathrm{O}$ polarization monitor setup as previously discussed.

\section{Simulations of shielding measurements}

Simulations using GEANT4 were performed for all of the different shielding configurations. In the simulations, neutrons were generated at the target according to the measured angular distribution with no shielding. This procedure ensured agreement between the measurements without shielding and the simulated results. It was assumed that the beam polarization did not degrade when passing through shielding materials between the target and the beam. This assumption is supported by the experimental measurement using copper shielding as shown in Fig. 4a, and is ultimately validated by the ability of the simulation to reproduce the measured values. Blocks of shielding were added in the simulation using the same geometries, dimensions, and orientations as in the experimental measurements. After the simulations were completed, one correction to the simulation was made. In simulations with shielding between the target and the beam, the simulated count rate was scaled down by the known attenuation factor of the shielding material [35], since this was not taken into account previously in the simulation.

\section{Experimental results: enrichment assay}

Figure 2 shows the experimental measurements of the polarization asymmetry as a function of the enrichment of a sample composed entirely of ${ }^{235,238} \mathrm{U}$. The solid line is an interpolation between two measurements: one on natural uranium and one on $93.7 \%$ enriched ${ }^{235} \mathrm{U}$. This interpolation is based on the measured asymmetries and $\sigma_{f} \bar{\nu}$ for each isotope using only those two data points, and is not fit to any of the other points. The dashed lines indicate the expected uncertainty in the interpolation.

The mixed-sample points of varying enrichments show good agreement with the interpolation. The mixed-sample points were taken with the new method 


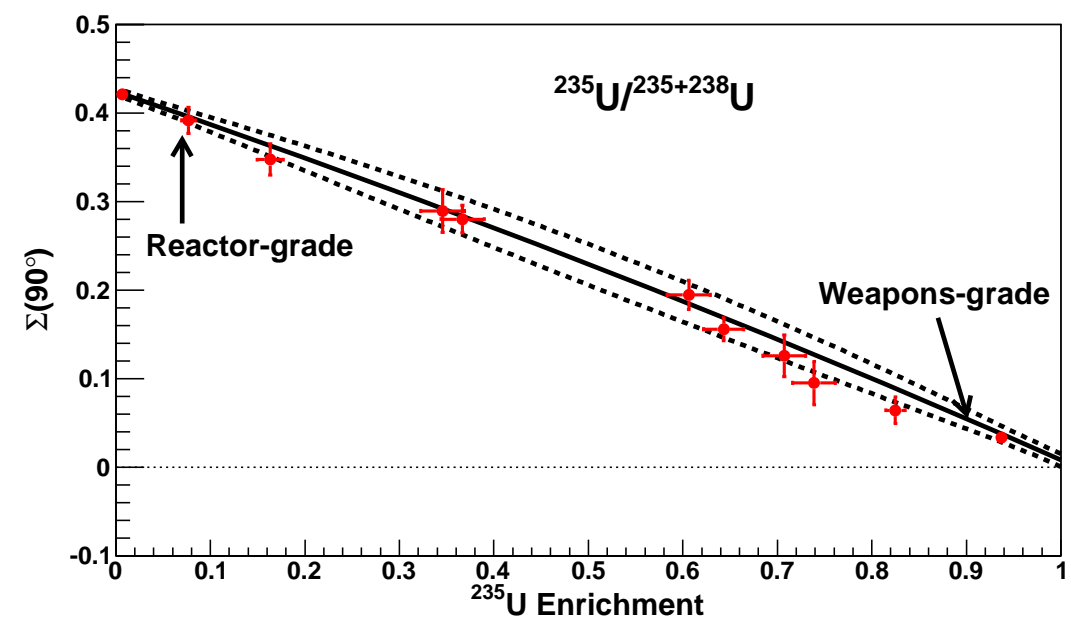

Figure 2: The polarization asymmetry at a scattering angle of $90^{\circ}$ is shown as a function of the enrichment of a sample composed entirely of ${ }^{235,238} \mathrm{U}$. The red points indicate experimental measurements. The error bars on the points are statistical and systematic combined. The solid black line is an interpolation between the two data points with smallest and largest enrichments. The dashed black lines indicate the combined statistical and systematic uncertainties on the interpolation.

of polarized $\gamma$-ray beam production, so their systematic uncertainties are larger than the other points. Despite the larger uncertainties, these measurements indicate that this technique can distinguish samples of different enrichments, and that the simple interpolation between the two points is reasonable.

A procedure was developed to fit the new experimental measurements to improve the interpolation and more reliably establish its uncertainty. First, a fit was performed using the same functional form as the interpolation function and only the statistical uncertainties in the data points. The statistical uncertainties in the fitted curve were calculated using the change in $\chi^{2}$ as the parameters were adjusted to neighboring values. Then, the points were moved systematically according to their correlated systematic uncertainties many times, and each time they were moved another fit was performed. The systematic uncertainty in the interpolation was calculated based on the variation in the interpolation 


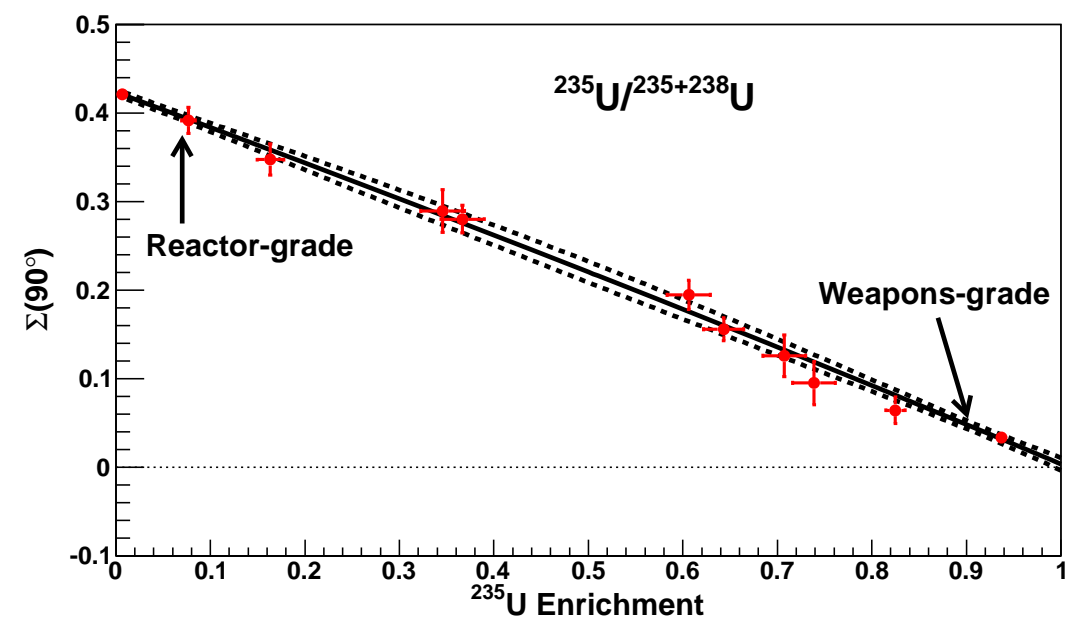

Figure 3: The polarization asymmetry at a scattering angle of $90^{\circ}$ is shown as a function of the enrichment of a sample composed entirely of ${ }^{235,238} \mathrm{U}$. The red points indicate experimental measurements. The error bars on the points are statistical and systematic combined. The solid black line is a fit to the data points. The dashed black lines indicate the combined statistical and systematic uncertainties on the fit.

over these many fits. Figure 3 shows the results of fitting the interpolation curve to all of the data points. The statistical uncertainty on the fit is negligible and the error bands are dominated by the systematic uncertainties.

\section{Experimental results: shielding}

Figure 4a shows the measured prompt neutron polarization asymmetry in the presence of the copper, lead, and steel shielding, and a subset of the results with polyethylene shielding. Overall, the polarization asymmetries are relatively robust to this amount of shielding. The largest reduction in the asymmetry is found using $2.54 \mathrm{~cm}$ of lead surrounding the target, which reduces the measured asymmetry by about $15 \%$.

It is clear that $2.45 \mathrm{~cm}$ of copper shielding between the beam and the target does not degrade the beam polarization (Fig. 4a). If the beam polarization were degraded by the shielding, the measured polarization asymmetry would be lower 


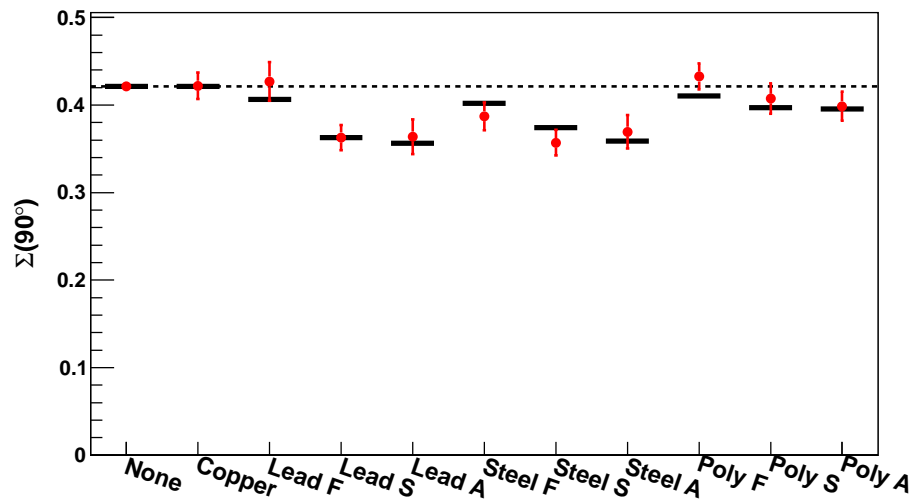

(a) The polarization asymmetry at a scattering angle of $90^{\circ}$

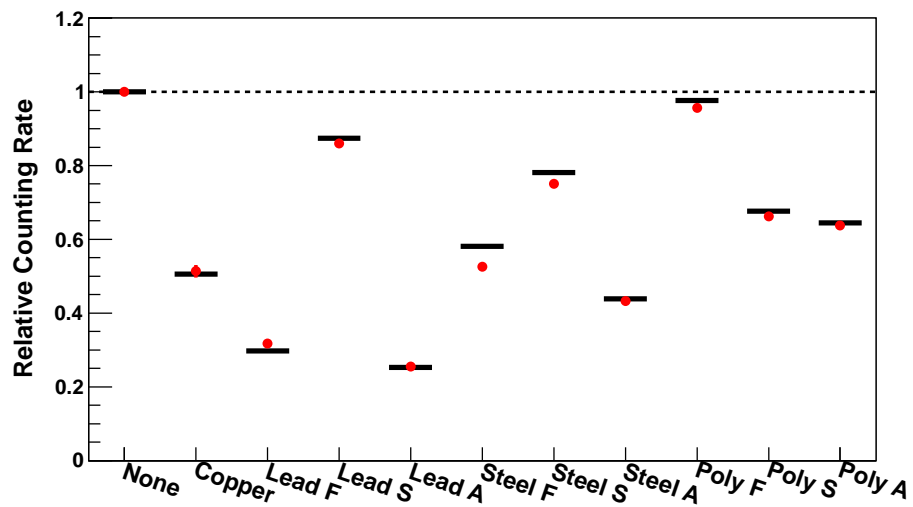

(b) The counting rate relative to the unshielded counting rate

Figure 4: The polarization asymmetry at a scattering angle of $90^{\circ}$ 4a and the counting rate relative to the unshielded counting rate $4 \mathrm{~b}$ are shown as a function of the location of copper, lead, steel, or polyethylene shielding. " $\mathrm{F}$ " indicates shielding between the target and the beam, "S" indicates shielding between the target and the detectors, and "A" indicates "F" and "S" shielding combined. All shielding materials were $2.54 \mathrm{~cm}$ thick, except for copper which was $2.45 \mathrm{~cm}$ thick and only placed in the front of the target. The red points are the experimental measurements, while the black lines are the simulated results. The error bars are statistical and systematic uncertainties combined. 


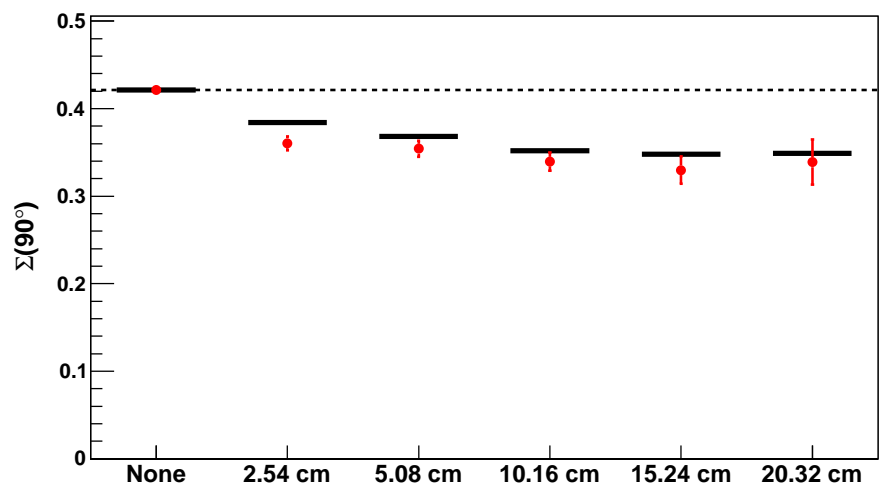

(a) The polarization asymmetry at a scattering angle of $90^{\circ}$

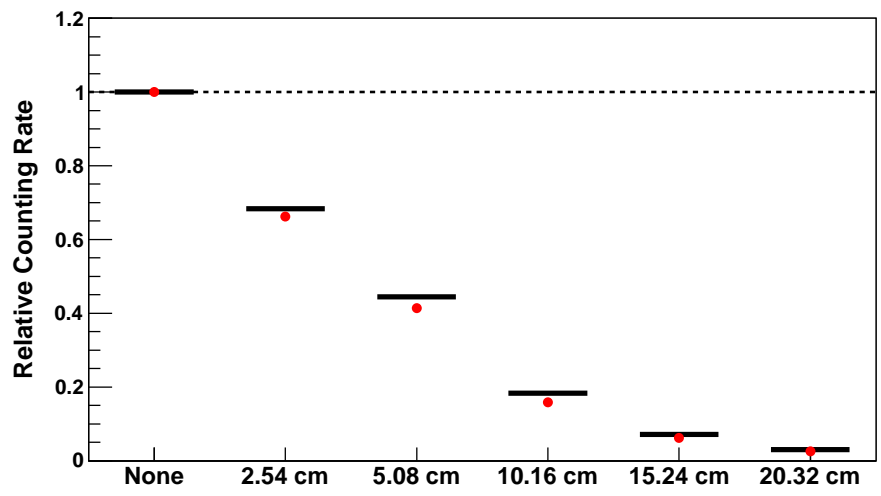

(b) The counting rate relative to the unshielded counting rate

Figure 5: The polarization asymmetry at a scattering angle of $90^{\circ}$ (5a) and the counting rate relative to the unshielded counting rate $[5 \mathrm{~b}$ ) are shown as a function of the thickness of high density polyethylene shielding placed on the sides of the target. The thickness of the shielding material is indicated in centimeters. The red points are the experimental measurements, while the black lines are the simulated results. The error bars are statistical and systematic uncertainties combined. 
than the measurement without shielding. The copper shielding was far upstream of the target, so no neutron multiple scattering off of the copper could occur within the neutron timing cuts. A small reduction in the measured asymmetry is predicted for the lead shielding in front of the target. This reduction is caused by multiple scattering of the neutrons off of the lead. In this configuration, the shielding was only $\sim 2 \mathrm{~cm}$ away from the target, so neutrons were able to reach the detector within the timing cuts after scattering off of the lead. All of the measurements shown with lead and polyethylene agree with the simulated results.

Figure $4 \mathrm{~b}$ shows the relative counting rate for the different shielding configurations. The count rate without any shielding was approximately 100 detected prompt neutrons per minute per detector averaged over the in-plane and outof-plane detectors. The largest reduction in the count rate is found with 2.54 $\mathrm{cm}$ of lead in front of the target. This amount of lead attenuates the beam by approximately $71 \%$. Steel in front of the target reduces the beam intensity by a smaller amount, and polyethylene in front of the target only slightly reduces the beam intensity. When the shielding is placed on the sides of the target, the opposite trend occurs; polyethylene provides the largest reduction, while lead provides the smallest reduction. All of the measured count rates with lead and polyethylene shielding show good agreement with simulated results.

There is a minor discrepancy present between the simulated and experimental results for the steel shielding. The measured count rates for steel in front of and on the sides of the target are slightly below the predicted values. The asymmetries for steel in front of the target and on the sides of the target are also lower than the predicted values. These slight disagreements could be caused by the uncertainty in the precise elemental composition of the steel. In the simulation, it was assumed the steel was 1018 cold rolled steel, which is approximately $99.1 \%$ iron, $0.2 \%$ carbon, and $0.7 \%$ manganese. However, a small admixture of other elements, such as tungsten, would reduce the count rate and asymmetry. No attempt was made to determine the precise composition of the steel sample that was used since the disagreements are small. 
Figure 5a shows the measured prompt neutron polarization asymmetry in the presence of additional high density polyethylene shielding. Overall, the polarization asymmetries are relatively robust to even large amounts of shielding; the largest reduction seen is from $20.3 \mathrm{~cm}$ of polyethylene on the sides of the target, which reduces the measured asymmetry by approximately 15\%. The agreement between the simulated and experimental results is good, though there appears to be a small, systematic difference between the simulated and measured results. This difference could be due to small inaccuracies in the GEANT4 ${ }^{1} \mathrm{H}(\mathrm{n}, \mathrm{n})$ cross sections or angular distributions. Overall, however, the agreement is good; the disagreement in most points is within the combined statistical and systematic uncertainties in the measurements.

The behavior of the asymmetry with increasing shielding is somewhat unexpected. For small shielding thicknesses, the polyethylene preferentially outscatters the prompt neutrons traveling in the horizontal plane. This out-scattering increases the yield measured in the vertical plane, which slightly decreases the polarization asymmetry. However, it appears that after approximately $10 \mathrm{~cm}$ of shielding is inserted, any additional shielding does not significantly reduce the asymmetry. One cause of this behavior is the dependence of the asymmetry on the neutron energy. The polyethylene shielding preferentially scatters the lower energy prompt neutrons $(\sim 1-2 \mathrm{MeV})$, since the ${ }^{1} \mathrm{H}(\mathrm{n}, \mathrm{n})$ cross section is larger there than at higher prompt neutron energies [36]. Therefore, as shielding is added, the higher energy neutrons constitute a larger fraction of the measured neutron yield. Previous measurements have shown that the higher energy prompt neutrons have larger polarization asymmetries than the lower energy neutrons [17]. This correlation may be one explanation of the observed behavior of the asymmetry for large amounts of shielding. Another cause of the observed dependence of the asymmetry is the specified timing window. Neutrons that scatter off of the outer layers of the shielding may not have time to reach a detector within the neutron timing cuts. Therefore, the outer layers of shielding may reduce the count rate observed in all detectors in an approximately equal way, which would not affect the polarization asymmetry. 
Figure 5b shows the relative counting rate for the different shielding configurations. It is clear from both the experimental and the simulated results that high density polyethylene can significantly scatter outgoing neutrons. Each 2.54 $\mathrm{cm}$ of polyethylene between the target and the detectors reduced the counting rate by approximately $37 \%$. Despite the significant reduction in count rate for large amounts of polyethylene shielding, the polarization asymmetry is relatively unchanged.

\section{Conclusions and future work}

A previously reported novel method of assaying SNM [1] has been tested. This method uses a linearly-polarized $\gamma$-ray beam to induce fission of actinide nuclei within a sample, and the asymmetry between the in-plane and out-ofplane prompt neutron yield is measured. An extended study of this new method involved using different combinations of multiple ${ }^{235} \mathrm{U}$ and ${ }^{238} \mathrm{U}$ foils to imitate samples of uranium with different enrichments. The agreement between the measured prompt neutron polarization asymmetries for these combined foils and the interpolation based on the measurements of highly enriched ${ }^{235} \mathrm{U}$ and natural uranium demonstrates the precision of this new technique. The accuracy of the interpolation was improved by fitting to these new results.

In addition to these experiments, novel tests were also performed with different shielding materials blocking either the beam from the target, the target from the detectors, or both of these scenarios combined. When shielding was placed between the beam and the target, no degradation in the beam polarization was observed for the materials and thicknesses used. Overall, the measured asymmetries and count rates agree with known $\gamma$-ray attenuation factors and simulations based on GEANT4 using the unshielded case as a baseline. The reduction in the measured asymmetries is typically less than $15 \%$ for shielding thicknesses of $2.54 \mathrm{~cm}$. It is interesting to note that the reduction in asymmetry makes non-fissile material look like fissile material, but it does not make fissile material look like non-fissile material. Therefore, in an active interrogation en- 
vironment where one is searching for shielded SNM, the shielding would increase the rate of false positives but not the rate of false negatives other than from the simple reduction in the count rate.

There still remain many challenges to using this technique in the field. One significant challenge is the difficulty in making a portable intense quasimonoenergetic linearly polarized $\gamma$-ray beam. Our current facility, $\mathrm{HI} \gamma \mathrm{S}$, has a footprint of several thousand square meters and is immobile. Therefore, $\mathrm{HI} \gamma \mathrm{S}$ or a HI $\gamma$ S-like facility for SNM detection or identification in the field is impractical. However, we hope that our measurements may be used to guide the development of future $\gamma$-ray beam sources, such as those made by Compton backscattering off of Wakefield accelerators [37], which have the potential to be more compact and mobile. Another potential challenge is the impact of neutron multiplication within the sample. The samples used here were all thin, rendering neutron multiplication negligible. However, in thicker samples of SNM, there may be significant neutron multiplication, which could reduce the measured polarization asymmetry. The impact of the neutron multiplication within a sample should be carefully studied in future experiments. A final challenge is the fact that all of the fissile nuclides studied have the same polarization asymmetry. Our previous results have demonstrated that the polarization asymmetry for all of the fissile actinides is close to zero at a beam energy of $6.1 \mathrm{MeV}$. Therefore, one disadvantage of using the polarization asymmetry to perform enrichment measurements is that different fissile actinides cannot be distinguished from each other.

In future experiments, it may be interesting to measure the prompt photofission neutron-neutron coincidence count rate on several actinides such as ${ }^{233,235,238} \mathrm{U}$, ${ }^{237} \mathrm{~Np}$, and ${ }^{239} \mathrm{Pu}$. It is possible that the average prompt neutron multiplicities will be different for these different isotopes, and therefore, the different isotopes may have different prompt neutron-neutron coincidence count rates in photofission. Additional information about the specific composition of a sample may be gained by simultaneously measuring both the polarization asymmetry and the neutron-neutron coincidence rate, and this additional information may be 
sufficient to determine the fissile versus non-fissile content in addition to the specific fissile isotopes within a sample. There would be no loss in sensitivity in simultaneously measuring the polarization asymmetry with the neutron-neutron coincidence counting rate, since the neutron-neutron coincidence counting rate measurements could be performed with the same detectors and beam configuration. Therefore, in future work, it may be useful to measure the neutron-neutron coincidence count rate in photofission of these fissile actinides.

\section{Acknowledgements}

The authors wish to thank the HI $\gamma \mathrm{S}$ staff for the development of the new technique of $\gamma$-ray beam polarization and for the $\gamma$-ray beams produced for this experiment. This work was supported in-part by DNDO, Academic Research Initiative (ARI) Grant \# 2010-DN-077-ARI46-02, DOE Grant \# DE-FG0297ER41033, DOE Grant \# DE-FG02-97ER41046, and by the DOE Office of Science Graduate Fellowship Program (DOE SCGF), made possible in part by the American Recovery and Reinvestment Act of 2009, administered by ORISEORAU under contract \# DE-AC05-06OR23100.

\section{References}

[1] J. M. Mueller, M. W. Ahmed, H. R. Weller, Nuclear Instruments and Methods in Physics Research Section A: Accelerators, Spectrometers, Detectors and Associated Equipment 754 (2014) 57-62.

[2] R. Berndt, E. Franke, P. Mortreau, Nuclear Instruments and Methods in Physics Research Section A: Accelerators, Spectrometers, Detectors and Associated Equipment 612 (2010) 309-319.

[3] C. T. Nguyen, J. Zsigrai, Nuclear Instruments and Methods in Physics Research Section B: Beam Interactions with Materials and Atoms 246 (2006) 417-424. 
[4] R. O. Korob, G. A. Blasiyh Nuño, Applied radiation and isotopes : including data, instrumentation and methods for use in agriculture, industry and medicine 64 (2006) 525-531.

[5] W. Zhang, J. Yi, P. Mekarski, K. Ungar, B. Hauck, G. H. Kramer, Applied radiation and isotopes : including data, instrumentation and methods for use in agriculture, industry and medicine 69 (2011) 904-907.

[6] J. Mihalczo, J. Mattingly, J. Neal, J. Mullens, Nuclear Instruments and Methods in Physics Research Section B: Beam Interactions with Materials and Atoms 213 (2004) 378-384.

[7] M. Rubio Montero, A. Martinsanchez, A. Carrascolourtau, Nuclear Instruments and Methods in Physics Research Section B: Beam Interactions with Materials and Atoms 213 (2004) 429-433.

[8] R. Pöllänen, T. Siiskonen, S. Ihantola, H. Toivonen, A. Pelikan, K. Inn, J. La Rosa, B. Bene, Applied Radiation and Isotopes 70 (2012) 733-739.

[9] R. T. Kouzes, E. R. Siciliano, J. H. Ely, P. E. Keller, R. J. McConn, Nuclear Instruments and Methods in Physics Research Section A: Accelerators, Spectrometers, Detectors and Associated Equipment 584 (2008) $383-400$.

[10] R. C. Runkle, D. L. Chichester, S. J. Thompson, Nuclear Instruments and Methods in Physics Research Section A: Accelerators, Spectrometers, Detectors and Associated Equipment 663 (2012) 75-95.

[11] K. A. Jordan, T. Gozani, Nuclear Instruments and Methods in Physics Research Section B: Beam Interactions with Materials and Atoms 261 (2007) $365-368$.

[12] T. Gozani, J. Bendahan, M. J. King, C. Brown, M. Elsalim, E. Elias, IEEE Transactions on Nuclear Science 60 (2013) 1118-1125. 
[13] E. B. Norman, S. G. Prussin, R.-M. Larimer, H. Shugart, E. Browne, A. R. Smith, R. J. McDonald, H. Nitsche, P. Gupta, M. I. Frank, T. B. Gosnell, Nuclear Instruments and Methods in Physics Research Section A: Accelerators, Spectrometers, Detectors and Associated Equipment 521 (2004) 608-610.

[14] A. Iyengar, E. Norman, C. Howard, C. Angell, A. Kaplan, J. Ressler, P. Chodash, E. Swanberg, A. Czeszumska, B. Wang, R. Yee, H. Shugart, Nuclear Instruments and Methods in Physics Research Section B: Beam Interactions with Materials and Atoms 304 (2013) 11-15.

[15] M. Johnson, C. Hagmann, J. Hall, D. McNabb, J. Kelley, C. Huibregtse, E. Kwan, G. Rusev, A. Tonchev, Nuclear Instruments and Methods in Physics Research Section B: Beam Interactions with Materials and Atoms 285 (2012) 72-85.

[16] B. Quiter, B. Ludewigt, V. Mozin, C. Wilson, S. Korbly, Nuclear Instruments and Methods in Physics Research Section B: Beam Interactions with Materials and Atoms 269 (2011) 1130-1139.

[17] J. M. Mueller, M. W. Ahmed, R. H. France, M. S. Johnson, H. J. Karwowski, L. S. Myers, J. Randrup, M. H. Sikora, M. C. Spraker, S. Stave, J. R. Tompkins, R. Vogt, H. R. Weller, C. S. Whisnant, W. R. Zimmerman, Physical Review C 89 (2014) 034615.

[18] J. M. Mueller, Prompt Neutron Polarization Asymmetries in Photofission of Isotopes of Thorium, Uranium, Neptunium, and Plutonium, Ph.D. thesis, 2013.

[19] V. N. Litvinenko, B. Burnham, M. Emamian, N. Hower, J. Madey, P. Morcombe, P. O'Shea, S. Park, R. Sachtschale, K. Straub, G. Swift, P. Wang, Y. Wu, R. Canon, C. Howell, N. Roberson, E. Schreiber, M. Spraker, W. Tornow, H. Weller, I. Pinayev, N. Gavrilov, M. Fedotov, G. Kulipanov, G. Kurkin, S. Mikhailov, V. Popik, A. Skrinsky, N. Vinokurov, B. Norum, A. Lumpkin, B. Yang, Physical Review Letters 78 (1997) 4569-4572. 
[20] H. R. Weller, M. W. Ahmed, H. Gao, W. Tornow, Y. K. Wu, M. Gai, R. Miskimen, Progress in Particle and Nuclear Physics 62 (2009) 257-303.

[21] Y. Wu, N. Vinokurov, S. Mikhailov, J. Li, V. Popov, Physical Review Letters 96 (2006) 224801.

[22] H. Arenhövel, Few-Body Systems 26 (1999) 43-98.

[23] H. Arenhövel, M. Sanzone, Photodisintegration of the Deuteron: A Review of Theory and Experiment, Springer-Verlag, Vienna, 1991.

[24] J. W. Chen, M. J. Savage, Physical Review C 60 (1999) 065205.

[25] G. Rupak, Nuclear Physics A 678 (2000) 405-423.

[26] B. D. Sawatzky, A Measurement of the Neutron Asymmetry in $d(g, n) p$ Near Threshold, Ph.D. thesis, 2005.

[27] M. A. Blackston, M. A. Ahmed, B. A. Perdue, H. R. Weller, B. Bewer, R. E. Pywell, W. A. Wurtz, R. Igarashi, S. Kucuker, B. Norum, K. Wang, J. Li, S. F. Mikhailov, V. G. Popov, Y. K. Wu, B. D. Sawatzky, Physical Review C 78 (2008) 1-9.

[28] W. Tornow, N. G. Czakon, C. R. Howell, A. Hutcheson, J. H. Kelley, V. N. Litvinenko, S. Mikhailov, I. V. Pinayev, G. J. Weisel, H. Witala, Modern Physics Letters A 18 (2003) 282-285.

[29] E. C. Schreiber, Measurement of a High-Intensity Gamma-Ray Beam and the Analyzing Power for $2 \mathrm{H}(\mathrm{g}, \mathrm{n}) \mathrm{p}$ Near Threshold, Ph.D. thesis, 2000.

[30] H. Arenhövel, private communication, 2014.

[31] G. Rupak, private communication, 2014.

[32] J. Allison, K. Amako, J. Apostolakis, H. Araujo, P. Arce Dubois, M. Asai, G. Barrand, R. Capra, S. Chauvie, R. Chytracek, G. Cirrone, G. Cooperman, G. Cosmo, G. Cuttone, G. Daquino, M. Donszelmann, M. Dressel, G. Folger, F. Foppiano, J. Generowicz, V. Grichine, S. Guatelli, 
P. Gumplinger, A. Heikkinen, I. Hrivnacova, A. Howard, S. Incerti, V. Ivanchenko, T. Johnson, F. Jones, T. Koi, R. Kokoulin, M. Kossov, H. Kurashige, V. Lara, S. Larsson, F. Lei, O. Link, F. Longo, M. Maire, A. Mantero, B. Mascialino, I. McLaren, P. Mendez Lorenzo, K. Minamimoto, K. Murakami, P. Nieminen, L. Pandola, S. Parlati, L. Peralta, J. Perl, A. Pfeiffer, M. Pia, A. Ribon, P. Rodrigues, G. Russo, S. Sadilov, G. Santin, T. Sasaki, D. Smith, N. Starkov, S. Tanaka, E. Tcherniaev, B. Tome, A. Trindade, P. Truscott, L. Urban, M. Verderi, A. Walkden, J. Wellisch, D. Williams, D. Wright, H. Yoshida, IEEE Transactions on Nuclear Science 53 (2006) 270-278.

[33] J. T. Caldwell, E. J. Dowdy, B. L. Berman, R. A. Alvarez, P. Meyer, Physical Review C 21 (1980) 1215-1231.

[34] J. T. Caldwell, E. J. Dowdy, R. A. Alvarez, B. L. Berman, P. Meyer, Nuclear Science and Engineering 73 (1980) 153.

[35] J. H. Hubbell, S. M. Seltzer, Tables of X-Ray Mass Attenuation Coefficients and Mass Energy-Absorption Coefficients from $1 \mathrm{keV}$ to $20 \mathrm{MeV}$ for Elements $\mathrm{Z}=1$ to 92 and 48 Additional Substances of Domestic Interest, 2004.

[36] E. Lomon, R. Wilson, Physical Review C 9 (1974) 1329-1335.

[37] S. Chen, N. Powers, I. Ghebregziabher, C. Maharjan, C. Liu, G. Golovin, S. Banerjee, J. Zhang, N. Cunningham, A. Moorti, S. Clarke, S. Pozzi, D. Umstadter, Physical Review Letters 110 (2013) 155003. 